\title{
Petrolio e politica nella decolonizzazione algerina: verso un network energetico europeo?
}

Pétrole et politique dans la décolonisation algérienne : vers un réseau européen de l'énergie?

Oil and Politics in Algerian Decolonization: Towards a European Energy

Network?

Marta Musso

(2) OpenEdition

Journals

Edizione digitale

URL: http://journals.openedition.org/cei/2925

DOI: $10.4000 /$ cei.2925

ISSN: 2260-779X

Editore

UGA Éditions/Université Grenoble Alpes

Edizione cartacea

Data di pubblicazione: 20 avril 2016

Paginazione: 141-156

ISBN: 978-2-84310-323-0

ISSN: $1770-9571$

Notizia bibliografica digitale

Marta Musso, «Petrolio e politica nella decolonizzazione algerina: verso un network energetico europeo?», Cahiers d'études italiennes [Online], 22 | 2016, online dal 01 janvier 2017, consultato il 26 mars 2021. URL: http://journals.openedition.org/cei/2925 ; DOI: https://doi.org/10.4000/cei.2925 


\title{
PETROLIO E POLITICA \\ NELLA DECOLONIZZAZIONE ALGERINA: \\ VERSO UN NETWORK ENERGETICO EUROPEO?
}

\author{
Marta Musso \\ University of Cambridge
}

\section{Il cartello del petrolio e la doppia dipendenza europea}

Nel 1956, la scoperta d'ingenti riserve di petrolio e gas naturale nel Sahara algerino fu salutata come l'inizio di una nuova era per la Francia - e per l'Europa in generale. Il deficit nel bilancio energetico rappresentava infatti un serio grattacapo per i governi e le imprese di tutto il continente, e del blocco occidentale in generale. La ricostruzione post-bellica si stava trasformando nel miracolo economico che avrebbe dato all'Europa il più alto tasso di crescita di sempre, e la paura che un arresto improvviso degli approvvigionamenti energetici facesse ricadere il vecchio continente in recessione cresceva in parallelo all'aumento delle importazioni di fonti energetiche dall'estero. Nell'ottobre del 1958, un report dell'Organizzazione per la cooperazione economica europea (Oece) avvertiva che entro gli anni Settanta il deficit energetico dell'Europa dei Sei avrebbe toccato i $200 \mathrm{mtce}^{\mathrm{I}}$. A preoccupare erano soprattutto le importazioni di petrolio, il cui consumo cresceva a ritmi vertiginosi e che si apprestava a superare il carbone come prima fonte di energia del mondo. Nel solo biennio 19541956, i consumi di petrolio in Europa erano aumentati di quasi il $20 \%$, e la crescita non sembrava destinata a rallentare nel breve o medio periodo ${ }^{2}$. Nell'era del petrolio, l'Europa era diventata un territorio dalle scarse risorse energetiche ${ }^{3}$. I paesi Oece avevano consumato in totale $78 \mathrm{I}$ mtce nel 1956; la produzione era stata di 615 mtce, grazie soprattutto al carbone e all'energia idroelettrica, mentre il deficit di 166 mtce era stato coperto con le importazioni di carbone dagli Stati Uniti e soprattutto di petrolio

I. Million Tons Coal Equivalent (tonnellate di carbone equivalente).

2. "Petroleum Press Service», aprile 1956.

3. È bene ricordare che i giacimenti del Mare del Nord furono messi in produzione solo negli anni Settanta. 
dal Medio Oriente 4 . Il report rilevava che queste importazioni non erano soltanto un fardello molto pesante sui bilanci europei, ma costringevano l'Europa a uno stato d'insicurezza costante e crescente.

I grandi produttori mondiali di petrolio negli anni Cinquanta erano innanzitutto gli Stati Uniti (36\% della produzione), seguiti da Medio

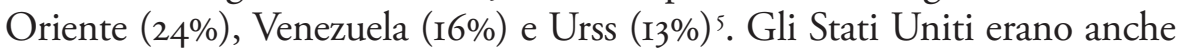
il primo consumatore, con quasi 500 milioni di tonnellate l'anno, e dal I948 erano diventati un paese importatore - uno shock per l'amministrazione Truman, che aveva imposto misure per limitare le esportazioni di petrolio all'estero e facilitare le importazioni dall'America Latina, più difendibili da un eventuale attacco sovietico ${ }^{6}$. La Russia riusciva invece a garantire la sufficienza energetica per sé e per i propri alleati, nonché la possibilità di esportare a basso prezzo. Il Medio Oriente, che possedeva il $63 \%$ delle riserve mondiali (contro il I2\% degli Stati Uniti), di lì a pochi anni avrebbe rimpiazzato l'America come primo produttore' ${ }^{7}$.

L'Europa, il terzo consumatore mondiale, riusciva invece a coprire poco più del $3,5 \%$ del proprio fabbisogno. Dopo il giro di vite sugli export di petrolio americano, il vecchio continente si era ritrovato a dipendere dal Medio Oriente per nove decimi del proprio fabbisogno ${ }^{8}$. Inoltre, le importazioni in Europa non erano gestite che in minima parte da compagnie europee. I paesi dovevano affidarsi alla gestione di alcuni grandi gruppi anglo-americani, che avevano creato una situazione di oligopolio nell'industria petrolifera internazionale e dato vita a un vero e proprio cartello per la coordinazione di produzione e distribuzione a livello mondiale, esercitando il controllo su più dell' $85 \%$ della produzione e distribuzione al di fuori di Stati Uniti e Urss?.

Le ragioni di questa dipendenza europea erano storiche e geografiche. L'industria petrolifera si era sviluppata negli Stati Uniti a partire dalla metà del XIX secolo, grazie alle ingenti risorse presenti sul territorio: i paesi

4. Archivi storici dell'Unione europea di Firenze, Conseil des ministres Cee et Euratom - 1958, Report OEEC, ottobre 1958.

5. Archivi storici dell'Unione europea di Firenze, Ceca Haute Autorité - CEABog Division Économie et Énergie. Cfr. Y. Delavesne, Annales des mines, juin I960.

6. Cfr. E. B. Kapstein, The Insecure Alliance: Energy Crises and Western Politics since 1944, Oxford, Oxford University Press, 1990; D. Yergin, The Prize, New York, Free Press, 2008; L. Maugeri, L'era del petrolio, Milano, Feltrinelli, 2006.

7. Cfr. H. Cattan, The Law of Oil Concessions in the Middle East and North Africa, Cambridge, Cambridge University Press, 1968.

8. Cfr. «Petroleum Press Service», aprile 1956; Archivi storici dell'Unione europea di Firenze, Conseil des ministres Cee et Euratom - 1958, Report OEEC, ottobre 1958.

9. E. Penrose, The Large International Firm in Developing Countries: The International Petroleum Industry, London, Allen \& Unwin 1968, p. 19. 
europei, privi della materia prima, si erano dovuti adattare al ruolo di second comer. Gli enormi costi iniziali e il fattore di rischio imposti dalla fase di esplorazione, uniti alla tendenza dell'industria all'integrazione orizzontale, avevano poi portato già agli inizi del XX secolo alla fusione delle compagnie petrolifere americane in pochi grandi gruppi che operavano a livello internazionale. Alla vigilia della prima guerra mondiale, le uniche due grandi compagnie europee erano la Royal Dutch Shell e la British Petroleum (Bp), fondate da imprenditori inglesi o olandesi che avevano la possibilità di operare nel Medio Oriente e nel Sud-Est asiatico. Insieme alle cinque principali compagnie americane (Standard Oil of New Jersey, Gulf Oil, Texaco, Standard Oil of California, Socony Mobil), questi gruppi controllavano interamente il mercato di raffinazione e distribuzione europeo ${ }^{\text {Io }}$. A partire dal 1928, queste compagnie avevano iniziato a coordinarsi in un cartello che non solo fissava le quote di produzione e i prezzi di vendita a livello internazionale, così da mantenere un flusso di approvvigionamento e di introiti stabili, ma si spartiva le aree di competenza sia nella ricerca del petrolio (per lo meno nell'area del Medio Oriente, che andava delineandosi come la più ricca), che nella distribuzione, con un controllo dei mercati pianificato. Come scrisse Paul Frankel, il più influente consulente dell'industria petrolifera degli anni Cinquanta e Sessanta ${ }^{\text {II, }}$ "le compagnie petrolifere sono diventate, quasi per distrazione (lo stesso stato d'animo che si presuppone sia stato responsabile della creazione dell'impero britannico), istituzioni internazionali che svolgono un ruolo vitale per tutti i paesi in cui operano» ${ }^{12}$. Il cartello agiva come un intermediario tra paese produttore e paese consumatore; assicurava che i flussi di petrolio fossero costanti e gli approvvigionamenti diversificati; e investiva nella ricerca di nuovi giacimenti e in tecnologie per l'industria ${ }^{\mathrm{I}}$. Fuori dal cartello, solo alcune compagnie indipendenti americane riuscivano a ritagliarsi zone di nicchia per l'estrazione e soprattutto di mercato. Queste compagnie, come scrisse Frankel, garantivano un 'alibi' al cartello: permettevano alcuni margini di competizione, ma se avevano troppo successo venivano acquisite dalle major a prezzi molto alti ${ }^{14}$.

Una terza categoria di compagnie petrolifere era rappresentata dalle imprese di Stato, a questa data quasi esclusivamente europee. Queste compagnie erano nate alla fine della prima guerra mondiale come tentativo dei

IO. Ibid.

II. G. Chandler, Obituary: Paul Frankel, «The Independent», 29 ottobre 1992.

I2. P. H. Frankel, Mattei: Oil and Power Politics, Westport, Praeger, 1966, p. IO2. Traduzione dell'autrice.

13. J. E. Hartshorn, Oil Companies and Governments, London, Faber \& Faber, 1967.

I4. P. H. Frankel, Mattei, cit., p. Io8. 
governi europei di colmare il proprio gap in un'industria ormai dominata dagli Stati Uniti ${ }^{15}$. Svolgevano soprattutto ricerche nel territorio nazionale o nelle colonie, oppure si concentravano nel settore downstream (raffinazione e distribuzione), comprando il greggio dal cartello e rivendendolo a prezzi competitivi, in modo da esercitare un minimo controllo sui prezzi locali. Il potere di queste imprese era molto limitato sia rispetto al cartello, sia rispetto alle indipendenti americane: nel 1956 una compagnia come la Standard godeva di un capitale di più di 900 miliardi di franchi, l'indipendente americana Sinclair di Ioo, mentre la più grande compagnia petrolifera pubblica europea, la Compagnie française des pétroles (Cfp), aveva un capitale di appena 34 miliardi di franchi ${ }^{16}$.

La Francia era il paese più attivo nel proporre una politica petrolifera nazionale. Oltre alla Cfp, che gestiva soprattutto una piccola quota di interessi nel Medio Oriente, nel corso degli anni i governi francesi avevano dato vita a diversi altri organi pubblici per promuovere la ricerca e la raffinazione del petrolio; il più importante era il Bureau de recherche de pétrole, fondato nel 1945 perché coordinasse la ricerca in tutta l'Unione francese. Un'altra importante compagnia petrolifera era quella italiana, l'Eni (Ente nazionale idrocarburi). A differenza della Cfp, che era partecipata dal governo francese solo per il 45\%, l'Eni era interamente pubblica, ed era stata fondata nel 1953 come gruppo ombrello che coordinasse e promuovesse tutte le fasi dell'industria petrolifera italiana ${ }^{17}$. Inizialmente, la compagnia si occupava di gestire le risorse di metano scoperte in Valle Padana e soprattutto di raffinare e distribuire il greggio acquistato dalla $\mathrm{Bp}$; ma fin dalla sua nascita aveva puntato a emulare la struttura delle major tramite una forte integrazione orizzontale e attività orientate al controllo diretto dei giacimenti petroliferi ${ }^{18}$. In un primo tempo il presidente dell'Eni, Enrico Mattei, imprenditore nel settore della chimica ed ex parlamentare democristiano, aveva cercato di entrare nell'orbita delle grandi compagnie del cartello chiedendo di partecipare al consorzio internazionale promosso dallo scià in Iran dopo il colpo di Stato che aveva deposto Mossadeq ${ }^{19}$. L'ambizione per una compagnia come l'Eni era di potersi ritagliare uno spazio di manovra sui giacimenti petroliferi simile

15. Cfr. L. Maugeri, L'era del petrolio, cit.; D. Yergin, The Prize, cit.

I6. Archivio storico Eni [AS Eni], Corrispondenza sulle ricerche nel Sahara. Per la storia della Cfp vedi L. Maugeri, L'era del petrolio, cit.; D. Pozzi, Dai gatti selvaggi al cane a sei zampe (1926-1967), Venezia, Marsilio, 2009 .

I7. D. Pozzi, cit.

I8. P. H. Frankel, Mattei, cit.; D. Pozzi, cit.

19. D. Pozzi, cit.; P. H. Frankel, cit. 
a quello della Cfp o delle indipendenti americane, in modo da assicurare all'Italia un accesso diretto alle risorse petrolifere. Tuttavia, dopo il rifiuto del cartello di far entrare l'Eni in Medio Oriente per motivi fondamentalmente relativi alla scarsa forza dell'Italia in politica estera, Mattei decise di cambiare completamente strategia ${ }^{20}$; grazie soprattutto alla sua intraprendenza personale, tra il 1956 e il 1960 l'Eni riuscì a siglare una serie di contratti che nel giro di pochi anni determinarono alcuni cambiamenti sostanziali nell'industria petrolifera mondiale.

\section{La formula Mattei e la crisi di Suez}

In un'intervista al giornalista francese Éric Laurent negli anni Settanta, il colonnello Gheddafi dichiarò che la sua politica petrolifera era stata influenzata soprattutto dagli eventi della guerra d'Algeria e dalla crisi di Suez ${ }^{21}$. La Libia, come del resto l'Algeria, fu uno dei paesi che implementarono un processo di nazionalizzazione dell'industria petrolifera più deciso, avendo come modello proprio Nasser e la prova di forza del 1956 contro le compagnie occidentali.

La crisi di Suez non fu infatti soltanto il canto della fenice degli imperi europei: mostrò anche quanto l'Europa fosse esposta nei confronti del mondo arabo a causa del petrolio. Nell'aprile del 1956, sei mesi prima dell'inizio della crisi, il passaggio di petroliere dal canale aveva stabilito il nuovo record di 38,4 milioni di tonnellate di greggio, un aumento del $67,3 \%$ rispetto all'anno precedente. Il petrolio era destinato soprattutto a Regno Unito (II,6 milioni di tonnellate), Francia (6,9 mt), Paesi Bassi $(4,5 \mathrm{mt})$, Italia (4 mt) e Stati Uniti (4 mt) ${ }^{22}$. Durante la chiusura del canale, la crisi energetica fu scongiurata grazie all'aumento di rifornimenti dagli Stati Uniti e dall'America Latina; inoltre le compagnie petrolifere si equipaggiarono velocemente con grandi petroliere per circumnavigare l'Africa ${ }^{23}$. In ogni caso, il prezzo del greggio sul mercato europeo aumentò di 2 dollari la tonnellata ${ }^{24}$, e Nasser poté sperimentare la teoria presentata nel suo pamphlet Filosofia della Rivoluzione: il petrolio era un'arma potente per il mondo arabo, un nervo vitale e scoperto per l'Occidente ${ }^{25}$. Per l'Fln e

\footnotetext{
20. Sulle vicende dell'Iran, v. P. H. Frankel, cit., p. 95.

2I. E. Laurent, La verità nascosta sul petrolio, Bologna, Nuovi Mondi Media, 2006, p. 97.

22. "Petroleum Press Service», ottobre 1956.

23. E. Laurent, cit., p. 72.

24. Ibid.

25. G. Nasser, The Philosophy of the Revolution, Il Cairo, S.O.P. Press, I952.
} 
gli altri leader indipendentisti, la riappropriazione delle risorse petrolifere divenne uno dei principali slogan nella lotta contro i poteri coloniali ${ }^{26}$.

Questo scenario di crescente tensione tra Occidente e mondo arabo fu sfruttato da Enrico Mattei per aprire importanti spazi di manovra al di fuori del cartello ${ }^{27}$. In quegli anni, la formula contrattuale più diffusa per le concessioni petrolifere era il cosiddetto 'fifty-fifty': oltre a pagare una royalty fissa per lo sfruttamento degli idrocarburi, le compagnie versavano metà degli introiti per la vendita del greggio ai paesi produttori. Anche se ciò significava enormi vantaggi economici in termini di gettito, questi erano consapevoli che il loro principale asset era fondamentalmente controllato da organi stranieri che possedevano la tecnologia e i mercati per gestire il petrolio, senza lasciare loro voce in capitolo. Dopo essere stato escluso dal consorzio, Mattei portò avanti nuovi negoziati con la compagnia nazionale iraniana (Nioc), basati sulla concessione all'Iran di maggiori capacità di controllo sulle proprie risorse ${ }^{28}$. L'accordo prevedeva che l'Eni ottenesse la concessione per i permessi di esplorazione gratis; in cambio, nel caso di ritrovamenti di petrolio in quantità commerciali, l'Eni e la Nioc avrebbero formato una joint-venture condivisa al 50\%. Il governo iraniano diventava quindi un partner alla pari. Il paese avrebbe controllato il 50\% della compagnia e ottenuto metà dei ricavi, come nella formula 'fifty-fifty'; in più avrebbe potuto disporre del $50 \%$ del petrolio estratto. Questa formula era innovativa per due motivi: il paese produttore accedeva agli aspetti gestionali dell'industria, e in più veniva reimmesso nell'industria petrolifera molto del capitale che prima veniva semplicemente pagato ai governi dei paesi produttori; azione che riduceva il capitale iniziale necessario per la messa in valore dei giacimenti e facilitava l'ingresso di nuovi operatori ${ }^{29}$.

Nella visione di Mattei, avere un middleman (la compagnia petrolifera) che trattava tra paese produttore e paese consumatore era uno spreco di denaro a favore della grande industria privata. Ridurre le compagnie allo stato di contractor e implementare una gestione dell'industria che fosse gestita direttamente dai due governi avrebbe reso possibile un risparmio per i paesi consumatori, con prodotti petroliferi meno cari; e avrebbe portato un vantaggio per i paesi produttori, che a fronte delle stesse royalty avrebbero controllato metà della produzione, diventando di fatto impren-

26. Cfr. D. Hirst, Oil and Public Opinion in the Middle East, London, Faber and Faber, 1966.

27. Cfr. M. Pirani, Poteva andare peggio, Milano, Mondadori, 2012.

28. Cfr. I. Tremolada, La via italiana al petrolio, Milano, L'Ornitorinco, $201 \mathrm{r}$

29. Ivi, p. II7. 
ditori petroliferi pur senza possedere la tecnologia o il capitale iniziale per avviare un'industria nazionale ${ }^{30}$.

Questa nuova tipologia contrattuale a joint-venture fu siglata per la prima volta in Iran nel marzo del 1957, e pochi mesi dopo in Egitto, paese in cui l'Eni lavorava già dal 1954 e in cui aveva continuato a operare anche dopo la cacciata di compagnie occidentali come la belga Petrofina ${ }^{31}$. Negli anni successivi, la cosiddetta 'formula Mattei' s'impose come il modello contrattuale più richiesto dai paesi produttori.

Negli stessi mesi dei negoziati con l'Iran e l'Egitto, la Francia si trovava a decidere come sviluppare le risorse del Sahara. Durante la crisi di Suez, Parigi aveva dovuto fare affidamento esclusivamente sul petrolio iraniano, trasportato dalle petroliere che passavano intorno all'Africa, ma che non arrivava a metà dei fabbisogni francesi. In queste circostanze, l'uso delle risorse algerine diventava fondamentale per la sicurezza francese ${ }^{32}$. In modo da mantenere pieno controllo sulle risorse, ma non avendo né la tecnologia né il capitale per procedere da sola alla messa in valore dei giacimenti, la Francia decise dopo un lungo dibattito di attuare una soluzione basata sulle joint-venture, in modo simile a quanto proposto da Mattei. Tuttavia, per poter considerare la Francia un paese produttore, bisognava considerare il Sahara una parte integrante del territorio francese.

\section{La riorganizzazione politica del Sahara}

La speranza di trovare petrolio nel Sahara era stata inseguita dalla Francia per decenni. Al 1956, quando gli sforzi furono ripagati, nell'Unione francese erano stati investiti 168 milioni di franchi. In larga maggioranza, questi finanziamenti provenivano dal governo francese $(68,3 \%)^{33}$; alcune compagnie straniere avevano progetti di ricerca nella Francia continentale, ma solo la Shell era presente nel Sahara, in parte per una politica protezionistica adottata dalla Francia, e in parte perché la zona non era particolarmente appetibile per gli investimenti esteri, viste le difficilissime condizioni climatiche e i risultati fino a quel momento incerti. La Francia

30. Ivi, p. II2.

3I. P. H. Frankel, cit., p. II7; D. Pozzi, cit.

32. Archives diplomatiques du ministère des Affaires étrangères [ADMAE] - Série : Affaires économiques et financières - Sous-série : Directeur - Wormser. In R. Cantoni e M. Musso, “Our Oil Won't Feed Our Slavery". Battles around Oil and Pipelines in Wartime Algeria. Lavoro presentato alla conferenza "Carbon Democracy and Revolution: Critical Perspectives from the Middle East and the Mediterranean», Durham University, I3 febbraio 2015 .

33. «Petroleum Press Service», luglio 1956. 
operava quindi in una condizione di semi-monopolio, grazie a strumentazioni acquisite da compagnie americane; ma i lavori erano in parte limitati dalla mancanza di personale esperto ${ }^{34}$.

Finalmente, nel gennaio 1956 le trivelle della Creps (il cui 35\% era della Shell) trovarono una grande quantità di petrolio a Edjelé (Ioo milioni di riserve provate), lungo il confine con la Libia; tra giugno e dicembre, la compagnia pubblica SN Repal fece le scoperte più importanti a Hassi Messaoud (6oo milioni di riserve provate) e soprattutto a Hassi R'Mel, con un giacimento di gas naturale della capacità di 2,4 miliardi $\mathrm{m}^{3}$, uno dei più grandi al mondo. Anche se l'ammontare delle riserve non poteva essere paragonato a quello del Medio Oriente, come alcune agenzie francesi suggerirono all'indomani delle scoperte ${ }^{35}$, il petrolio e il gas sahariano erano abbastanza per poter coprire completamente il deficit energetico della Francia, e in larga parte quello dell'Europea dei Sei ${ }^{36}$.

La notizia della scoperta fu accolta con entusiasmo in tutto il continente; tuttavia, passato il momento di euforia, il problema della viabilità tecnica, economica e politica della creazione di un'industria petrolifera nel Sahara si presentò più arduo del previsto. L'elefante nella stanza era, naturalmente, la guerra in Algeria; già nel 1956 le attività di esplorazione nel Nord del paese avevano dovuto essere abbandonate, e la costruzione di oleodotti verso la costa avrebbe richiesto la militarizzazione dell'intera tratta $^{37}$. Consapevoli che i dipartimenti del Nord stavano sfuggendo dal controllo francese, il Brp e il governo si attrezzarono per separare de facto i territori del Sud con un nuovo assetto giurisdizionale ${ }^{38}$. Agli inizi del 1957, il Sud dell'Algeria fu organizzato in due nuovi dipartimenti (Oasis e Saoura), sotto il controllo di un nuovo Ministero del Sahara alle dipendenze dirette di Parigi. Contemporaneamente, venne finalmente avvallato un progetto su cui si discuteva da qualche anno: l'Organisation commune des régions sahariennes (Ocrs), un organo intergovernativo tra paesi confinanti con il Sahara che aveva l'obiettivo di portare avanti progetti di sviluppo nell'area ${ }^{39}$. In questo modo, mentre l'amministrazione politica veniva messa sotto l'egida diretta della métropole, l'aspetto economico veniva gestito insieme

34. Ibid.; R. Cantoni e M. Musso, cit.

35. AS Eni, Corrispondenza sull'Algeria.

36. Archivi storici dell'Unione europea di Firenze, Uwe Kitzinger and Noël Salter Documentation, Investimenti dei paesi Europei nell'industria petrolifera al $I^{\circ}$ gennaio I962; Archivi storici dell'Unione europea di Firenze, Conseil des ministres Cee et Euratom - I958.

37. «Petroleum Press Service», luglio 1956.

38. "Petroleum Press Service», giugno 1956

39. L. Blin, L’Algérie, du Sahara au Sahel, Paris, L’Harmattan, I990, p. 89; J. Loyrette, Le Code pétrolier saharien, Paris, Librairie générale de droit et de jurisprudence, I96I.

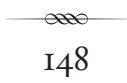


ad altri governi africani, nella speranza per Parigi che questo aiutasse a rinforzare l'asse di alleanze con gli altri paesi dell'Unione francese ${ }^{4}$.

Un secondo problema di tipo giuridico, ma anche fortemente politico, riguardava il rinnovo delle concessioni assegnate nel quinquennio precedente, che avrebbero dovuto essere rinnovate tra il 1957 e il 1958 in un quadro completamente mutato. Inizialmente, il governo socialista di Guy Mollet e il Brp capitanato da Pierre Guillaumat optarono per una politica pressoché autarchica. Nell'aprile del 1957, il ministro degli Esteri francese Christian Pineau dichiarò che il governo non avrebbe permesso a compagnie non francesi di ottenere nuovi permessi nell'area ${ }^{41}$. Dopotutto, erano stati in larga parte gli investimenti statali a ritrovare gli idrocarburi, e non si voleva che questi andassero ad arricchire compagnie straniere senza che il governo potesse esercitare controllo sulla sicurezza degli approvvigionamenti e sui prezzi. Inoltre, per la Francia il Sahara rappresentava l'occasione di sviluppare una propria industria petrolifera, con l'acquisizione di competenze e di lavoro specializzato francese. Le motivazioni erano quindi assimilabili a quelle degli altri paesi produttori: con la differenza che la Francia era anche un paese fortemente consumatore. Inoltre, il governo francese aveva motivi ben precisi di diffidenza nei confronti delle compagnie del cartello. Nel febbraio 1956, appena un mese dopo il ritrovamento di petrolio a Edjelé, la Legione straniera aveva scoperto delle prospezioni non autorizzate da parte della Standard Oil of New Jersey in Libia ${ }^{42}$. Ma soprattutto, nell'ottobre del 1956, quando Ben Bella venne arrestato, la stampa francese riportò che erano stati ritrovati addosso al leader indipendentista una serie di documenti su accordi segreti tra l'Aramco (il braccio della Standard Oil of California in Arabia Saudita) e l'Fln per future concessioni in cambio di aiuto militare. Nonostante le smentite dell'ambasciata americana, Mollet non negò ufficialmente le accuse, usando anzi queste speculazioni contro i fautori di una open policy nel Sahara ${ }^{43}$.

Tuttavia, nonostante la Francia partisse da una miglior competenza tecnica e un maggior capitale a disposizione rispetto ai paesi produttori tradizionali, la realtà del Sahara rendeva difficile fare a meno delle compagnie straniere, soprattutto americane. $\mathrm{Al}$ di là delle dure condizioni lavorative nel pieno del deserto, della distanza dei giacimenti dall'Europa (con un

40. L. Blin, cit.

4I. B. Bagnato, L'Italia e la guerra d'Algeria (I954-1962), Soveria Mannelli, Rubbettino, 20I2, p. 309-3IO.

42. Archives nationales d'Outre-Mer, Aix-en-Provence [ANOM - FM], Affaires algériennes (I873/1964). R. Cantoni e M. Musso, cit.

43. National Archives and Records Administration [NARA - RG 59], Central Decimal Files, I955-I959, French Africa. In R. Cantoni e M. Musso, cit. 
mare in mezzo) e del problema sicurezza causato dalla guerra, c'era anche e forse soprattutto il fatto che la rete di distribuzione del petrolio in Europa fosse controllata pressoché esclusivamente dalle compagnie del cartello. Quando il primo greggio sahariano iniziò ad arrivare alla Francia nel 1958, una crisi di sovrapproduzione dovuta alla concorrenza delle indipendenti americane e dell'Eni aveva già abbassato i prezzi. Rendere il greggio del Sahara competitivo non era quindi facile, anche perché gli alti costi di estrazione e trasporto avrebbero reso conveniente il petrolio francese solo a pieno regime di produzione. La Francia provò a chiedere alla Cee che fosse data per decreto la priorità agli approvvigionamenti dall'Africa; ma l'Europa rifiutò per paura che sostituire l'oligopolio americano con un monopolio francese avrebbe non solo fatto impennare i prezzi, ma danneggiato le relazioni con gli Stati Uniti ${ }^{44}$.

La presenza delle compagnie americane era quindi fondamentale, nonostante le presunte trame dell'Aramco, per ragioni sia tecniche che politiche. Dopo un vivace dibattito intervallato dal crollo della Quarta Repubblica, il nuovo code pétrolier approvato nell'ottobre del 1958 cercò così di salvaguardare il primato francese e allo stesso tempo di aprire all'estero. Il codice stabiliva che, per poter partecipare allo sfruttamento delle risorse, una compagnia straniera avrebbe dovuto stabilire una filiale in Francia e creare una joint-venture con un ente francese in cui fosse prevista anche la partecipazione del Brp. Inoltre, la compagnia avrebbe dovuto apportare capitale tecnico e personale specializzato, non solo finanziario, e avrebbe avuto l'obbligo di trasmettere tutti i dati rilevati nella fase di ricerca al $\mathrm{Brp}^{45}$. In compenso, il numero massimo di quote della joint-venture che la compagnia straniera poteva controllare veniva alzato dal $49 \%$ al 50\%, e furono introdotte una serie di agevolazioni fiscali. Questo sistema era pensato per favorire in modo particolare la partnership con le compagnie indipendenti americane che, al contrario delle majors, non controllavano grandi giacimenti nel Medio Oriente e avevano quindi un reale interesse a investire nel Sahara. Inoltre, almeno nelle speranze del governo francese, il loro minore peso politico e diplomatico non avrebbe reso possibile trattare con l'Fln; al contrario, avrebbero fatto pressione su Washington per proteggere lo status quo ${ }^{46}$.

44. AS Eni, Corrispondenza sull'Algeria.

45. R. Cantoni e M. Musso, cit.

46. AS Eni, Corrispondenza sull'Algeria. 


\section{Dall'Eurafrica all'Eurafrigas e la controproposta di Mattei}

Oltre che all'industria indipendente americana, la Francia guardava con speranza alla Comunità europea come partner per lo sfruttamento delle risorse sahariane. L'atteggiamento francese, però, mostrava numerose contraddizioni nei confronti della nascente Cee. Da una parte, Parigi non poteva fare a meno dell'aiuto europeo per trovare un mercato per i propri idrocarburi; dall'altra, il BRP rifiutò di creare un ente di livello europeo per la gestione dell'industria petrolifera del Sahara, preferendo negoziati bilaterali con le singole compagnie o i singoli paesi.

Per quanto riguarda il petrolio, come si è visto, la Cee rifiutò di concedere un trattamento privilegiato alla Francia; attorno al gas naturale, invece, si creò un notevole interesse. Con la parziale eccezione dell'Italia, l'Europa non aveva all'epoca alcun mercato per il gas; ma le quantità presenti nel solo giacimento di Hassi R'Mel, con un potenziale di produzione di 75 mtce di metano l'anno, potevano bastare a coprire completamente il deficit energetico francese (di 43,86 mtce nel 1956) e metà di quello europeo (I66 mtce). Inoltre, la mancanza di un network distributivo preesistente, come invece era per il petrolio, apriva alla possibilità di creare un sistema integrato che abbassasse notevolmente i costi di approvvigionamento. In un progetto presentato al Consiglio europeo nell'aprile del I958, la commissione energia propose la creazione di un unico network di distribuzione che interessasse non solo l'Europa mediterranea e centrale, ma anche il Maghreb, come prima concreta infrastruttura realizzata in uno spazio eurafricano ${ }^{47}$. Un network unificato avrebbe potuto portare notevoli vantaggi per l'integrazione europea: dividendo i costi iniziali e pianificando un sistema di trasporto per l'approvvigionamento di tutte le aree, i prezzi del gas sarebbero diventati estremamente competitivi rispetto a quelli del petrolio ${ }^{4}$.

Per quando riguarda la parte africana del progetto, il Consiglio concordava che il Maghreb avrebbe dovuto essere il primo e naturale utilizzatore delle risorse. Il laburista britannico Hilary Marquand ricordò che non si poteva separare l'Eurafrigas dal problema più generale dello sfruttamento delle risorse mondiali da parte degli occidentali, e che lo sviluppo dei campi petroliferi in Medio Oriente stava causando profondo malcontento tra le popolazioni arabe; bisognava quindi associare i governi africani già nelle

47. Report del Consiglio europeo sull'uso del gas nel Sahara del Nord, Conseil des ministres Cee et Euratom 1958, Archivi storici dell’Unione europea di Firenze. Traduzione dell'autrice.

48. Ibid. 
fasi di elaborazione del piano ${ }^{49}$. L'Africa però, citata in apertura e chiusura del progetto per ricordare l'importanza di usare il metano per promuovere lo sviluppo locale e facilitare le negoziazioni diplomatiche con l'Africa del Nord, spariva dalla parte tecnica del rapporto, se non per precisare che il livello dei consumi nel Maghreb era troppo basso allo stato attuale per investire nella creazione di una rete di trasporto, mentre l'Europa aveva bisogno urgente del gas sahariano, «vitale per il futuro dell'Europa, dell'Africa e del mondo libero» ${ }^{\circ}$. Per il momento quindi, il piano si sarebbe concentrato su come trasportare in gas in Europa; il Maghreb avrebbe dovuto aspettare l'avvio di un processo di sviluppo.

Il progetto Eurafrigas venne approvato dal Consiglio all'unanimità, e alla fine del 1958 l'ex segretario di Stato francese per l'industria Maurice Lemaire creò un gruppo di lavoro per gli studi preliminari. In particolare, la Francia era interessata a una partnership con l'Eni. In teoria, infatti, la compagnia avrebbe potuto essere un ottimo alleato per la Francia nel Sahara: un ente pubblico, con una visione europeista, che stava aprendo nuovi spazi per l'industria al di fuori del cartello. I contatti tra l'Eni e il Brp erano iniziati già nel 1956, prima della crisi di Suez, e a più riprese tra il 1957 e il 1958 il Bureau aveva fatto alcune proposte all'Eni per una partnership secondo la formula 49-51\%, e poi 50-50\% dopo la revisione del code pétrolier ${ }^{51}$. L'Eni aveva declinato tutte le offerte, con la motivazione ufficiale di preferire la creazione di una compagnia europea rispetto a joint-venture bilaterali. In realtà, Mattei non voleva compromettersi con i francesi, convinto che prima o poi l'indipendenza dell'Algeria sarebbe stata inevitabile. Un'idea condivisa dalle compagnie, straniere e francesi, presenti nel Sahara, molte delle quali portarono avanti contatti con l'Fln e il Gouvernement provisoire de la République algérienne (Gpra) in modo da garantire i propri interessi ${ }^{{ }^{2}}$. Ma anche se l'Fln non perdeva occasione di dichiarare che ogni accordo con i francesi sarebbe stato annullato all'indomani dell'indipendenza ${ }^{53}$, nessuno credeva che le compagnie sarebbero state cacciate una volta avviati i lavori di estrazione; e nel frattempo era

49. Discussione del Consiglio d'Europa sul rapporto Eurafrigas, Conseil des ministres Cee et Euratom - I958, Archivi storici dell'Unione europea di Firenze. Traduzione dell'autrice.

50. Report OEEC su uso gas Nord Sahara, Conseil des ministres Cee et Euratom - 1958, Archivi storici dell'Unione europea di Firenze. Traduzione dell'autrice.

5I. AS Eni, Corrispondenza sulle ricerche nel Sahara.

52. Oltre all'episodio delle speculazioni sull'Aramco, cfr. le dichiarazioni di Mario Pirani in B. Bagnato, L'Italia e la guerra d'Algeria (I954-I962), cit.

53. «El Moudjahid», ottobre I956. Vedi anche: Anep, El Moudjahid, un journal de combat (I956-I962), Rouiba, Anep, 2011. 
importante riuscire ad accedere alle concessioni prima che venissero tutte assegnate $^{54}$. Ma Mattei, a differenza delle altre compagnie, godeva di un primato diplomatico nel mondo arabo in generale e con Nasser (grande sostenitore dell'Fln) in particolare. Invece di un accordo con la Francia, sfruttò la propria posizione per concludere nel luglio del 1958 un accordo con il Marocco per la creazione della Società marocchino-italiana dei petroli (Somip) per la ricerca e la produzione di idrocarburi e per la costruzione di una raffineria a Mohammediass. Questo contratto suscitò le proteste del governo francese, che accusò l'Italia di aver estromesso la Francia dal Marocco $^{56}$; in effetti, l'obbiettivo nemmeno troppo velato di Mattei era sfruttare il processo di decolonizzazione per aumentare l'influenza politica ed economica dell'Italia in Maghreb a scapito della Francia, ritagliando per il paese 'un posto al sole' di primo piano sulla scena mediterranea.

Al tempo stesso però, l'azione dell'Eni fu caratterizzata da maggiore trasparenza rispetto alle strategie seguite dalle altre compagnie petrolifere nel Sahara. Forte dell'accordo con il Marocco infatti, nel novembre dello stesso anno Mattei fece una controproposta alla Francia per la creazione di una compagnia eurafricana che potesse essere la base non solo di progetti come l'Eurafrigas, ma un primo passo verso futuri negoziati con l'Algeria ${ }^{57}$. Invece di una joint-venture italo-francese per il Sahara, Mattei propose la creazione di tre compagnie: una in Tunisia, una in Marocco e una in Algeria. In Tunisia la joint-venture sarebbe stata controllata al 50\% dal governo tunisino, al $15 \%$ dall'Italia e al 35\% dalla Francia. In Marocco, dove l'Eni aveva appena siglato il contratto, proponeva di cedere il $15 \%$ della propria quota Somip alla Francia. Riguardo all'Algeria, Mattei propose come soluzione-ponte durante la guerra di creare una joint-venture Italia-Francia, con un eventuale coinvolgimento della Spagna (passaggio obbligato per la costruzione di oleodotti o gasdotti); con la pace, il 50\% delle quote sarebbe passato al governo indipendente algerino. Inoltre, finita la guerra, queste tre compagnie avrebbero potuto fondersi in un unico consorzio gestito pariteticamente dai paesi del Maghreb e dai paesi europei.

54. Pirani, cit.; NARA - RG 59, Central Decimal Files, I955-1959, French Africa, in R. Cantoni, Enemy Underground, PhD thesis, University of Manchester, 20I4. Cfr. anche le dichiarazioni dell'ambasciatore Quaroni in B. Bagnato, cit., p. 316.

55. Eni, Enistoria - inizia il viaggio / I958 - I pionieri del nucleare, <http://enistoria.eni.com/it/inizia-ilviaggio/I958.html>.

56. AS Eni, Corrispondenza sulle ricerche nel Sahara, Report: partecipazione italiana sfruttamento del petrolio sul Sahara, 20 ottobre 1958.

57. AS Eni, Corrispondenza sulle ricerche nel Sahara, Report incontro Mattei-Ollian a Roma per possibile collaborazione Francia-Italia-paesi arabo-sahariani sul problema dello sfruttamento delle risorse sahariane, 20 novembre 1958 . 
In questo modo, anche se la zona africana era in una posizione svantaggiata per i consumi, i governi del Maghreb avrebbero potuto accedere al controllo sulla rete europea, trattenendo il necessario per il proprio fabbisogno tramite quote che sarebbero accresciute man mano che più ampi processi di industrializzazione fossero stati messi a punto ${ }^{58}$. Nella proposta, Mattei sottolineava che questa soluzione avrebbe permesso all'Europa e alla Francia di conservare il Sahara senza l'insostenibile peso politico della guerra. Mattei si offriva infine, se la Francia avesse considerato i suoi interessi abbastanza tutelati, di prendere contatto con Algeria, Tunisia e Marocco e dare inizio ai negoziati ${ }^{59}$. La Francia rispose in maniera negativa alla proposta dell'Eni; nella crisi del 1958 non c'erano ancora i presupposti per poter intavolare i negoziati di pace. Dopo il rifiuto francese, l'Eni continuò in ogni caso i propri negoziati in Tunisia, con la creazione nel 1960 della la Société italo-tunisienne d'exploitation pétrolière (Sitep) ${ }^{60}$. I toni di Mattei si fecero sempre più apertamente anticoloniali, come in occasione del discorso per la firma dell'accordo con la Tunisia, in cui unì idealmente la lotta dell'Fln contro la Francia a quella dell'Eni contro il cartello petrolifero in un unico tema.

Io sono qui per rispondere al vostro appello d'investimenti e per aiutarvi nella lotta contro il sottosviluppo. Non ho paura della guerra in Algeria. Non ho paura della decolonizzazione. Io credo alla decolonizzazione non solo per ragioni morali di dignità umana ma per ragioni economiche di produttività. [...] Esiste una condizione coloniale quando il giuoco della domanda e dell'offerta per una materia prima vitale è alterato da una potenza egemonica: anche privata, di monopolio e di oligopolio. Nel settore del petrolio questa potenza egemonica-oligopolistica è il cartello ${ }^{6 \mathrm{I}}$.

Nello stesso periodo, l'Eni reclutò un ex giornalista dell'Unità, Mario Pirani, perché seguisse in segreto i negoziati con il Gpra, di stanza a Tunisi, per i futuri progetti dell'Eni in Algeria. Solo tre anni più tardi, con i negoziati di Lugrin e poi con quelli di Évian, venne trovato un accordo che riconosceva piena indipendenza all'Algeria e con una formula di gestione condivisa delle risorse petrolifere. Si trattò però, per quanto riguarda gli idrocarburi, di una tregua fragile, caratterizzata soprattutto dalla volontà algerina di estromettere la Francia. Invece dell''organisme mixte' che avreb-

58. AS Eni, Intervista a Mario Pirani, giugno 201 .

59. AS Eni, Corrispondenza sulle ricerche nel Sahara.

6o. Eni, Enistoria - inizia il viaggio / I96o - Il Marocco ci mette una pietra sopra, <http://enistoria.eni.com/ it/inizia-il-viaggio/ı6o.html>.

6I. Discorso pronunciato a Tunisi in occasione degli accordi italo-tunisini, 9-Io giugno 1960. In E. Mattei, Scritti e discorsi (1945-1962), Milano, Rizzoli, 2012. 
be dovuto curare la cogestione delle risorse, fu la Sonatrach, la compagnia di stato algerina, a prendere in mano il controllo sull'industria petrolifera, affossando i più ampi progetti di sviluppo su cui si era speculato negli anni del precedenti.

\section{Conclusioni}

Le ipotesi di storia controfattuale, non potendo essere verificate in alcun modo, lasciano spesso il tempo che trovano. Sebbene con l'obbiettivo a breve termine di aumentare l'influenza italiana in Nord Africa a scapito della Francia, il progetto proposto da Mattei merita tuttavia di essere indagato per il potenziale innovativo nei rapporti tra paesi sviluppati e sottosviluppati. Soprattutto, la creazione di una compagnia realmente mista e di un network distributivo unico tra Europa e Africa avrebbero potuto sia mettere l'Europa al riparo dallo shock petrolifero del 1973, sia proteggere i paesi del Maghreb dal controshock degli anni Ottanta, in cui il crollo degli idrocarburi causò gravissimi crisi economiche che furono, come nel caso proprio dell'Algeria, seguite da guerre. Anziché adottare un progetto di lungo respiro e in chiave internazionale, la gestione francese della messa in valore dell'industria petrolifera sahariana fu invece caratterizzata soprattutto dalla fretta di consolidare le proprie posizioni in modo che potessero resistere sia all'indipendenza algerina che all'oligopolio del cartello. L'industria petrolifera del Sahara non avvicinò l'Europa all'Africa se non per sporadici accordi intervallati da difficili negoziazioni; né vi fu traccia, all'epoca o nei quarant'anni successivi, di una politica energetica europea. Un'occasione persa, vista la tendenza dell'industria petrolifera a favorire gli accordi di lungo termine e la coordinazione: il cartello delle multinazionali, come poi l'Opec, non era che una risposta a queste esigenze.

È vero che Enrico Mattei, nel voler eliminare la presenza del middleman, non aveva considerato che gli accordi diretti tra Stati sarebbero stati molto più difficili proprio per la mancanza di un mediatore che si occupasse di questioni puramente economiche. Il presidente dell'Eni pensava che si sarebbe creato un sistema più giusto, in cui i governi avrebbero stabilito accordi mutualmente benefici e in cui gli alti margini di guadagno sarebbero andati ai rispettivi Stati invece che a gruppi privati. Ma i governi dei paesi produttori, diventando gestori diretti delle risorse petrolifere, diventarono anche competitor delle compagnie dei paesi consumatori. Inoltre, la mancanza di un operatore economico neutro rispetto ai processi politici lasciò spesso ogni contrattazione in balia di scontri politici 
sia interni che internazionali ${ }^{62}$. La storia dell'industria petrolifera francese in Algeria successiva agli anni qui analizzati, conclusasi con l'allontanamento delle compagnie pubbliche a favore dei privati, ne è una prova ${ }^{63}$.

Ma la proposta dell'Eni alla Francia nel 1958 non era quella di una semplice joint-venture tra un paese produttore e uno consumatore; era il progetto per una compagnia autenticamente internazionale, e quindi un organo super partes. Una compagnia che avrebbe permesso agli africani di entrare nel sistema distributivo europeo così come gli europei erano presenti nel sistema distributivo africano. Un progetto eccessivamente ambizioso in un'area dilaniata dalla guerra e in un paesaggio appena postcoloniale; ma che presentava una formula innovativa di gestione degli idrocarburi, e che voleva essere autenticamente eurafricano. 